\title{
Real-time decoding of 5 finger movements from 2 EMG channels for mixed reality human-computer interaction
}

\author{
Eric J. McDermott ${ }^{12^{*}}$, Thimm Zwiener ${ }^{3}$, Ulf Ziemann ${ }^{1}$, Christoph Zrenner ${ }^{1}$ \\ ${ }^{1}$ Department of Neurology and Stroke, and Hertie Institute for Clinical Brain Research, Eberhard Karls \\ University Tübingen, Germany \\ 2 International Max Planck Research School, and Graduate Training Center of Neuroscience, Tübingen, \\ Germany \\ ${ }^{3}$ Cartography and GIS Research Group, Department of Geography, Vrije Universiteit Brussel, Brussels, \\ Belgium \\ * Corresponding author: EricJamesMcDermott[at]gmail.com
}

\section{Keywords:}

EMG; Virtual Reality; Augmented Reality; Mixed Reality; Human-computer Interaction; $\mathrm{HCl}$; Machine Learning; Classification; k-Nearest Neighbors; Sensor Fusion

\section{Abstract}

The search for optimized forms of human-computer interaction $(\mathrm{HCl})$ has intensified alongside the growing potential for the combination of biosignals with virtual reality $(V R)$ and augmented reality $(A R)$ to enable the next generation of personal computing. At the core, this requires decoding the user's biosignals into digital commands. Electromyography (EMG) is a biosensor of particular interest due to the ease of data collection, the relatively high signal-to-noise-ratio, its non-invasiveness, and the ability to interpret the signal as being generated by (intentional) muscle activity. Here, we investigate the potential of using data taken from a simple 2-channel EMG setup to differentiate 5 distinct movements. In particular, EMG was recorded from two bipolar sensors over small hand muscles (extensor digitorum, flexor digitorum profundus) while a subject performed 50 trials of dorsal extension and return for each of the five digits. The maximum and the mean values across the trial were determined for each channel and used as features. A k-nearest neighbors $(\mathrm{kNN})$ classification was performed and overall 5 -class classification accuracy reached $94 \%$ when using the full trial's time window, while simulated real-time classification reached $90.4 \%$ accuracy when using the constructed kNN model $(k=3)$ with a $280 \mathrm{~ms}$ sliding window. Additionally, unsupervised learning was performed and a homogeneity of $85 \%$ was achieved. This study demonstrates that reliable decoding of different natural movements is possible with fewer than one channel per class, even without taking into account temporal features of the signal. The technical feasibility of this approach in a real-time setting was validated by sending real-time EMG data to a custom Unity3D VR application through a Lab Streaming Layer to control a user interface. Further use-cases of gamification and rehabilitation were also examined alongside the integration of eye-tracking and gesture recognition for a sensor fusion approach to $\mathrm{HCl}$ and user intent. 


\section{Introduction}

Electromyography (EMG) is the standard methodology used to measure muscle activity (Bigland-Ritchie 1981), and it has been used in controlling prosthetic devices (Castellini et al. 2009; Cipriani et al. 2008; Kiguchi et al. 2004; Kim et al. 2019), robotics (Fukuda et al. 2003; Gruver 1994) and rehabilitation (Graupe et al. 1982; Kawase et al. 2017). William Putnam was one of the first to describe a real-time EMG system (1993), and now, EMG and other wrist-based modalities are being explored as an input device for virtual, augmented, mixed, and extended reality applications for both research (Aung et al. 2011; Chowdhury et al. 2013; Jung et al. 2013; Kwon et al. 2020; Viriyasaksathian et al. 2011) and commercial purposes (Pezent et al. 2019; Young et al. 2019). The addition of biosignal-based agency within these mixed realities can help close the action-confirmation loop and offer critical feedback to improve performance and increase immersion (Sigrist et al. 2013). Finger movements already serve as a base control input in learned motor patterns such as mouse clicking, keyboard typing, and button pushing, yet it remains a technological challenge how such biologically based behaviors can be seamlessly integrated into the virtual world without manipulatable tools (i.e., mouse and keyboard) in order to increase human agency and immersion.

One approach at this "integration" requires a reliable translation of different movement-based actions into distinct classes of input based on the biological signals gathered from the actions, in a machine learning based procedure known as "decoding". The first part consists of extracting a small number of features from the raw EMG signal, while the second part consists of determining the probability that the observed features were generated by a specific movement. In supervised learning, this requires use of a classification algorithm which is calibrated using a subset of the total data as training data. A number of previous studies have presented methodologies implementing this approach (Englehart et al. 2001; Hudgins et al. 1993; Zardoshti-Kermani et al. 1995), some achieving up to $93 \%$ on an online four-class problem (thumb, index, middle, hand closed) using 4 distinct EMG channels (one channel per digit) (Tsenov et al. 2006).

Here, we investigate the potential of achieving high classification accuracy of individual finger movements with minimal hardware cost and demands while using a simple feature extraction method. Specifically, we use only 2 distinct EMG channels and do not consider temporal features of the signal. Furthermore, we explore the potential to integrate this into a portable off-the-shelf real-time system by utilizing $\mathrm{C}++$ based serial streaming from an Arduino Uno board (http://www.arduino.cc/), into a Python based model (http://www.python.org/), and then into a C\# based Unity3D application (http://www.unity3d.com/) using a Lab Streaming Layer (Kothe et al. 2012). The use of EMG in real-time allows for versatile interaction with computer interfaces, and can be further optimized by combining it with eye-tracking as a second-level check of user intention (i.e., gaze fixation). This concept of sensor fusion can also be further extended by the inclusion of integrated gesture recognition given advances in computer vision. 


\section{Experimental Methods}

Data was collected through a serial stream coming from the Arduino Uno board via USB connection. A commercially available EMG sensor ("Myoware Muscle Sensor") was used to extract the envelope of the EMG signal at 10-bit resolution, sampled at a default rate of $70 \mathrm{~Hz}$. Sensors were placed at both the extensor digitorum (ED) and flexor digitorum profundus (FD). No filters were applied. Data for this singlesubject proof-of-principle study was collected from one of the experimenters. First, the subject placed their arm and hand to rest comfortably on a table, with the wrist flat and the elbow padded. Then, the subject was instructed in a short calibration phase to perform a 'maximum' muscle contraction for each of the two EMG electrode sites (i.e., active wrist extension for ED, and active wrist flexion for FD), and a 'minimum' muscle contraction (i.e., be completely relaxed). These values then defined the subject's range of output values, and the subsequently recorded data were normalized accordingly within these limits. No incoming data values exceeded the established minimum or maximum.

The main measurement consisted of 50 repetitions of the sequential active dorsal extension of each individual digit and subsequent return to the baseline rest position over a 2-second window, in an up-anddown motion, with 2-second pauses in between each movement. The beginning of each trial was cued by a countdown presented on a computer monitor.

To account for reaction time delays (on average between 150-400ms according to previous studies (Kutas et al. 1980; Ladd 1911; Murakami 2010)), and other edge effects, the first 200ms and last 200ms of each trial were removed, resulting in trials $1600 \mathrm{~ms}$ long. Then, for each electrode, the max and mean value of the EMG envelope for each of the 50 trials was calculated, as well as for sliding windows of $70,140,280$, 560 , and 1020 milliseconds of each trial. The feature space was explored by a grid search of different variations of these features until the optimal feature set was found for both complete trial prediction and real-time prediction. Logistic, support vector machine (SVM), k-nearest neighbors (kNN), and DBSCAN (Hahsler et al. 2019) classifiers were compared and tested.

To validate the approach in a real-time setting, the resulting machine learning model was saved, and then a serial read was called from Python, where the incoming single time point data was transformed into a sliding average and sliding max. These newly created variables were then used as input features. Next, the resultant output class was directly sent to Unity3D using a Lab Streaming Layer. The virtual environment was designed and navigated with the HTC Vive (http://www.vive.com), furthermore the integrated eyetracking from SRanipal (https://developer.vive.com/resources/vive-sense/sdk/vive-eye-tracking-sdksranipal/) was used to affirm gaze locking on an object, and if confirmed, EMG activity could then manipulate the object or otherwise interface with it accordingly. To further extend the amount of use cases for this paradigm, additional gesture recognition using the HTC Vive Hand Tracking SDK 
(https://developer.vive.com/resources/vive-sense/sdk/vive-hand-tracking-sdk/) was also applied in particular instances, such as to instantiate interactable user interfaces.

\section{Results}

\section{Supervised Learning Approach}

To visualize the relationship between EMG sensor activation and finger extension, a kernel density estimation with a Gaussian kernel was performed to obtain the probability density of the distribution of EMG sensor values received during the trials for each of five digits, for each EMG sensor, as shown in Fig 1.
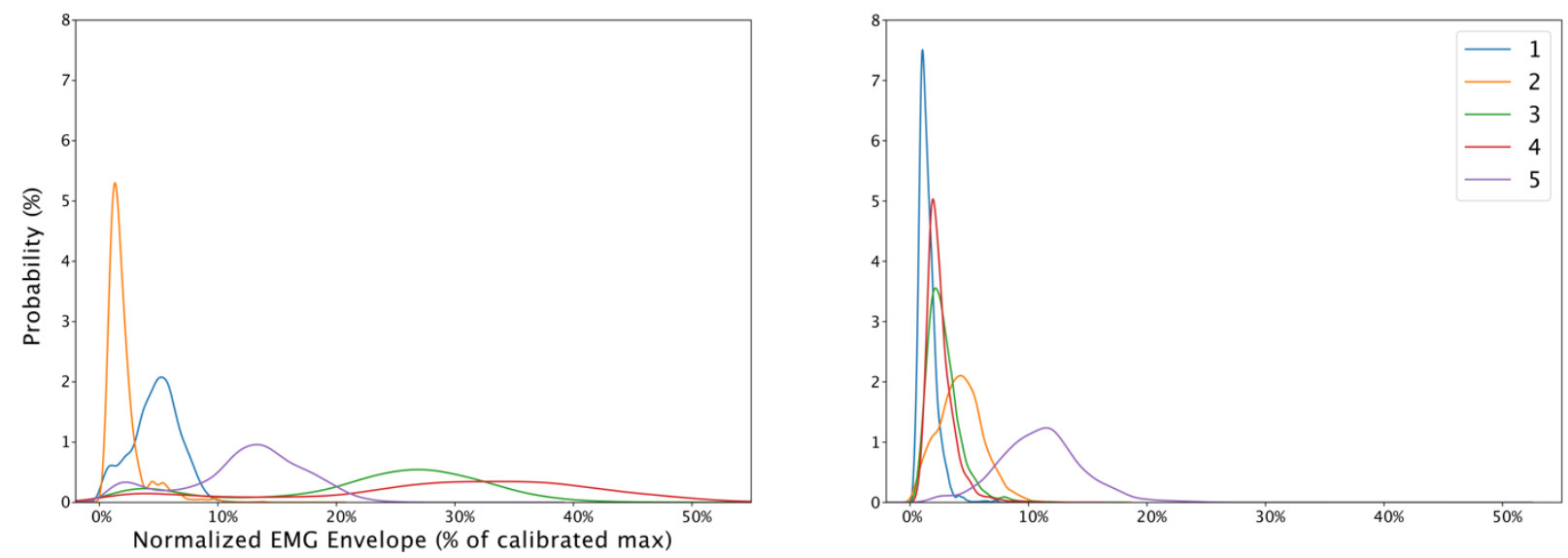

Fig 1: Kernel density estimation using Gaussian kernel for muscle activations in the ED (left panel) and FD (right panel) muscles. The horizontal axis corresponds to the EMG envelope (as a percentage of the maximum calibrated strength). Digits are numbered and color coded in sequential order starting with the thumb.

For the subsequent classification, the 'mean' and 'max' for each of the two EMG channels during each digit's movement was extracted, obtaining four features, see Fig 2a. Substantial separation between classes can be seen in this four-dimensional feature-space by visual inspection. However, because determining the max or mean value for a whole trial requires the entire time window, this classification can only be performed after and not during the trial, thereby restricting its usage for real-time applications. In order to enable classification during finger extension, the same 'mean' and 'max' features were also extracted from a sliding window with a length of $280 \mathrm{~ms}$ ( 20 data points), see Fig $\mathbf{2 b}$, which is near the upper limit of latency still enabling a responsive user experience for basic applications (Henderson 2001). 

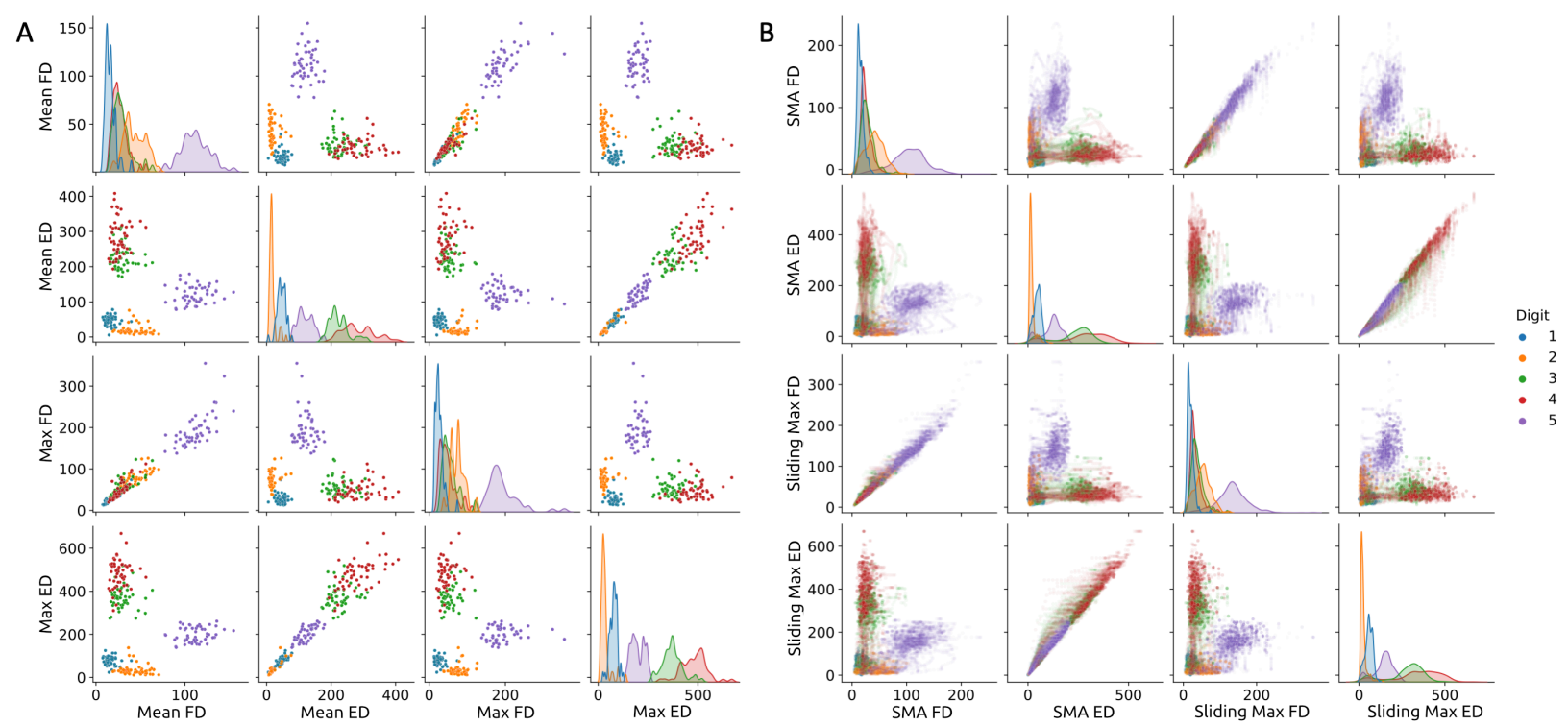

Fig 2: Pairwise plot of all four features against each other ('mean' and 'max' from both EMG channels) using (A) data from the entire trial $(1600 \mathrm{~ms})$ and $(B)$ data from a $280 \mathrm{~ms}$ long sliding window, during the trial. SMA; smoothed moving average. In panel B, due to the increased number of data points, the transparency level was adjusted to avoid complete occlusion of overlapping points. The diagonal cells show the probability distribution of the respective feature for each digit.

Using a kNN classifier with $k=3$, a decoding accuracy of $94 \%$ was achieved using the total window length, and a $90.4 \%$ accuracy using the sliding window length $280 \mathrm{~ms}$. A confusion matrix using this sliding window was computed to investigate the source of classification errors (see Fig 3), which showed substantial confusion decoding middle and ring finger movements (digits 3 and 4).
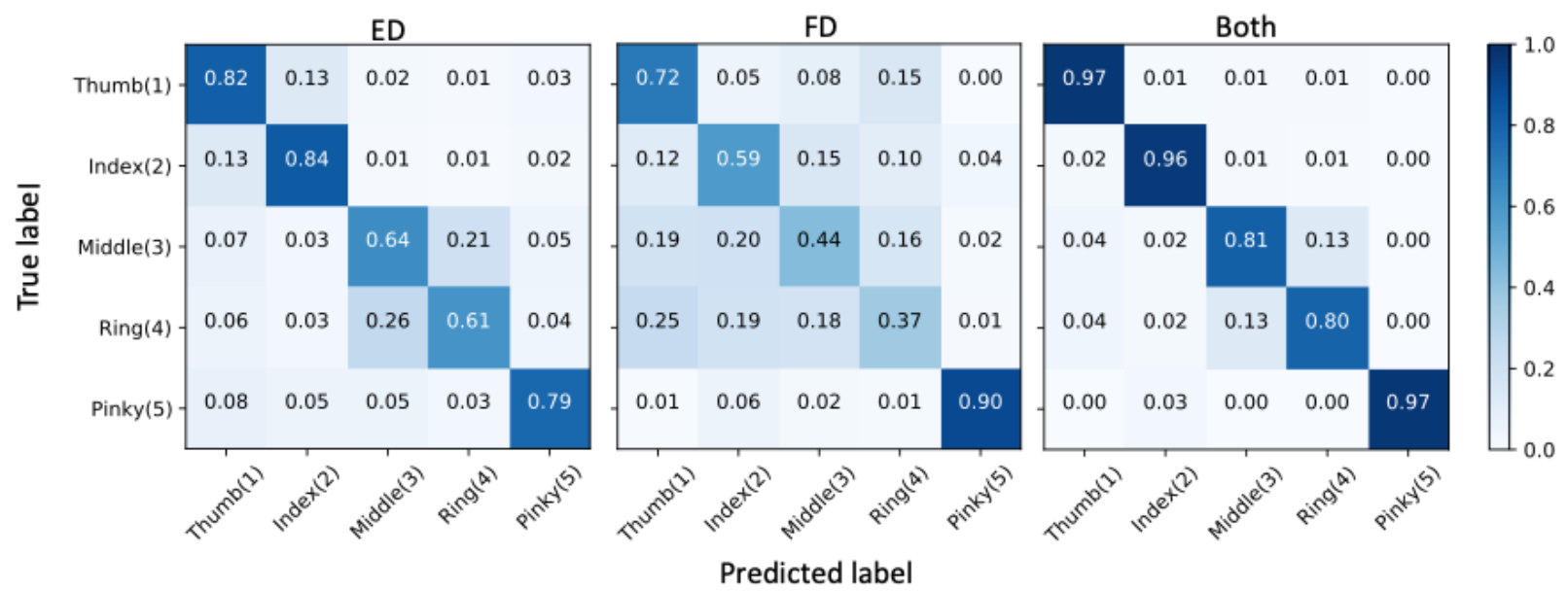

Fig 3: Normalized confusion matrix for each muscle independently, as well as together (ED, FD, Both). Features used were the 'max' and 'mean' taken from a 280ms sliding window, and tested throughout the $1600 \mathrm{~ms}$ of the trial using $\mathrm{kNN}$ with $k=3$. 


\section{Unsupervised Learning Approach}

We also performed an exploratory investigation into the separability of the five digit classes with an unsupervised learning approach. The DBSCAN classifier with standard settings (epsilon $=0.5$, minimum samples $=10$ ) and $k$-Means (with the number of clusters pre-defined as 5 ) were compared; with $k$-Means being superior when using the 'max' feature only ( $79 \%$ of labels correct), and when using the combination of 'max' and 'mean' (85\% of labels correct), Fig 4.
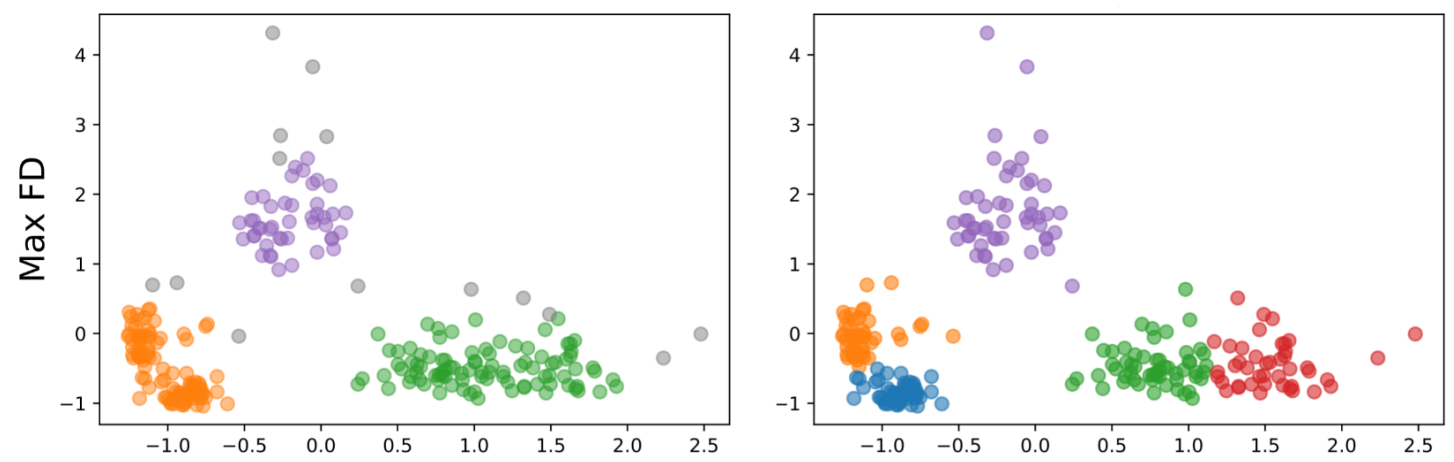

Digit

- 1

- 2

- 3

- 4
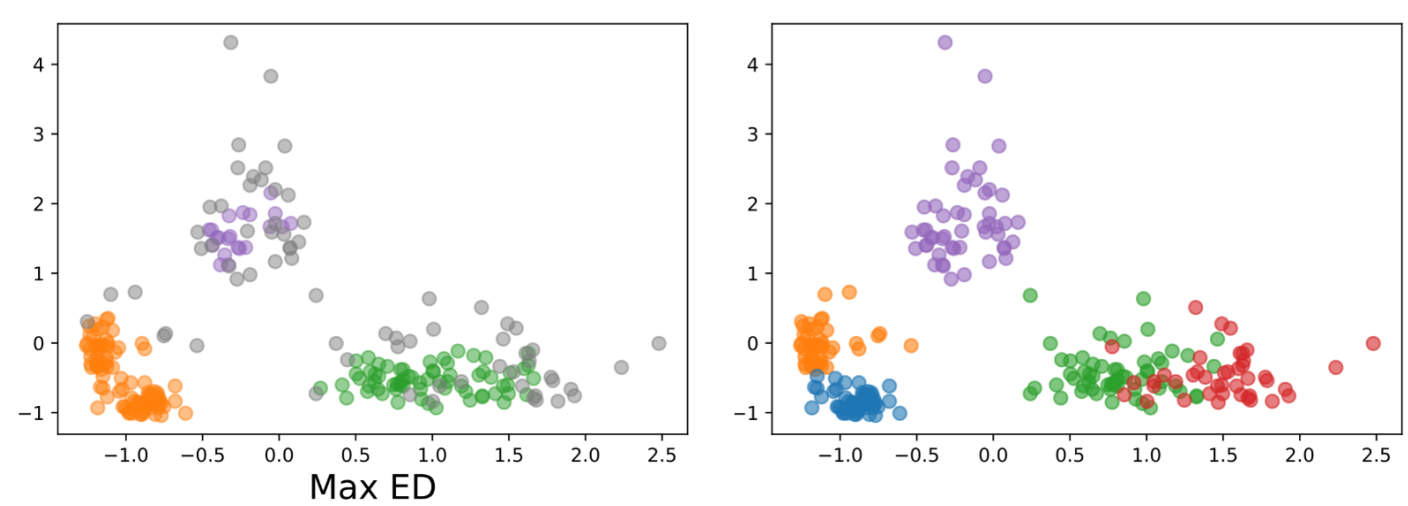

Fig 4: Comparison of unsupervised cluster based machine learning algorithms, DBSCAN (left) and k-Means with 5 clusters (right). (Top) classifications using only the 'max' of each channel as feature input. (Bottom) classifications using 'max' and 'mean' as feature inputs (only 'max' plotted). The $x$ and y-axis scales are arbitrary values. Grey points in DBSCAN represent uncategorized data points (i.e., outliers that are too far from a central node).

\section{Discussion}

The aim of this simple proof-of-concept study was to investigate the feasibility of an EMG decoding approach for use in mixed reality real-time applications. We demonstrate that five different movements corresponding to finger extensions of each individual digit can be decoded with an accuracy $>90.4 \%$ using only two EMG channels, $280 \mathrm{~ms}$ sliding windows, and a standard machine learning pipeline that uses simple 'mean' and 'max' features with a k-nearest neighbors classifier. The entire pipeline is implemented on lowcost off-the-shelf hardware and open-source software. We illustrate how this approach can be used to 
incorporate an EMG-based interface for human interaction with a virtual environment, such as controlling a user interface (see Supplementary Material 1), interacting with a 'smart' home (see Supplementary Material 2), or in gamified rehabilitation.

One limitation of this study is that we simply chose extension movements of the individual digits, whereas the repertoire of movements could be expanded to include various hand and finger signs and gestures. Also, the set of movements was not optimized for decoding, for example, the confusion between the middle and ring fingers is likely due to the physiological overlap and resultant "enslaving" effects of innervation, as documented by Zatsiorsky et al. (2000). This "enslaving" effect can also be visualized when closer examining the results of the unsupervised machine learning algorithms, as they too failed to adequately separate the two overlapping groups of data from the third and fourth digits. When excluding these two digits, our real-time method reaches closer to $97 \%$ accuracy.

Additionally, though we purposefully chose to use only two EMG sensors, we did not optimize the placement of the sensors for the task other than choosing the main muscles for finger extensions (the ED), and contractions (the FD). To this point, the sensor on the ED produces across-the-board higher classification accuracies than the sensor on the FD. This very well could be due to the experimental design, as dorsal extension of the fingers does call for full activation of the ED, yet the subsequent return to a 'flat'-handed home position would only partially activate the FD (full activation would require more-so closing the digits into a fist). Likely, attuned placement of the sensors in line with specific task requirements, a higher number of EMG sensors, or using a sensor fusion approach by including additional sensors such as computer vision and/or accelerometers would enable an even higher classification accuracy. It also might be necessary when considering applications spanning a greater range of movement classes, especially when they are largely overlapping. Furthermore, using only a single subject is an obvious limitation, but given the nature of similarity in innervation between people (Yu et al. 2004), and that this was a proof-of-principle demonstration, is sufficient for this purpose.

The main conclusion of this study in light of the above limitations, a satisfactory decoding accuracy is feasible in a real-time setting. Moving toward biosignal-controlled devices transitions from the conceptual framework of the use of computers as a tool toward one that is an intuitive extension of oneself and further expands the capabilities of the human experience. We look forward to the next generation of humancomputer interfaces that utilize biosignals to enable intuitive and frictionless embodiment for professional, recreational, and medical applications. 
bioRxiv preprint doi: https://doi.org/10.1101/2021.09.28.462120; this version posted September 30, 2021. The copyright holder for this preprint (which was not certified by peer review) is the author/funder, who has granted bioRxiv a license to display the preprint in perpetuity. It is made available under aCC-BY-NC-ND 4.0 International license.

\section{Supplementary Material}

Supplementary Material 1: video "EJM_UI", showing EMG / eye tracking to trigger UI menu

Supplementary Material 2: video "EJM_SmartHome", showing EMG / eye tracking / gesture recognition to interact with "smart home" 
bioRxiv preprint doi: https://doi.org/10.1101/2021.09.28.462120; this version posted September 30, 2021. The copyright holder for this preprint (which was not certified by peer review) is the author/funder, who has granted bioRxiv a license to display the preprint in perpetuity. It is made available under aCC-BY-NC-ND 4.0 International license.

\section{Declarations}

\section{Funding}

This project has received funding from the German Federal Ministry of Education and Research (BMBF, grant agreement number 13GW0213A).

\section{Conflict of Interest}

E.J.M., T.Z., U.Z. and C.Z. report no conflicts of interest. 


\section{References}

Aung YM, Al-Jumaily A Rehabilitation exercise with real-time muscle simulation based EMG and AR. In: 2011 11th International Conference on Hybrid Intelligent Systems (HIS), 2011. IEEE, pp 641-646

Bigland-Ritchie B (1981) EMG/force relations and fatigue of human voluntary contractions Exercise and sport sciences reviews 9:75-118

Castellini C, Van Der Smagt P (2009) Surface EMG in advanced hand prosthetics Biological cybernetics 100:35-47

Chowdhury A, Ramadas R, Karmakar S (2013) Muscle computer interface: A review ICoRD'13:411-421

Cipriani C, Zaccone F, Micera S, Carrozza MC (2008) On the shared control of an EMG-controlled prosthetic hand: analysis of user-prosthesis interaction IEEE Transactions on Robotics 24:170-184

Englehart K, Hudgin B, Parker PA (2001) A wavelet-based continuous classification scheme for multifunction myoelectric control IEEE Transactions on Biomedical Engineering 48:302311

Fukuda O, Tsuji T, Kaneko M, Otsuka A (2003) A human-assisting manipulator teleoperated by EMG signals and arm motions IEEE transactions on robotics and automation 19:210-222

Graupe D, Salahi J, Kohn KH (1982) Multifunctional prosthesis and orthosis control via microcomputer identification of temporal pattern differences in single-site myoelectric signals Journal of Biomedical Engineering 4:17-22

Gruver WA (1994) Intelligent robotics in manufacturing, service, and rehabilitation: An overview IEEE Transactions on Industrial Electronics 41:4-11

Hahsler M, Piekenbrock M, Doran D (2019) dbscan: Fast density-based clustering with R Journal of Statistical Software 91:1-30

Henderson T Latency and user behaviour on a multiplayer game server. In: International Workshop on Networked Group Communication, 2001. Springer, pp 1-13

Hudgins B, Parker P, Scott RN (1993) A new strategy for multifunction myoelectric control IEEE transactions on biomedical engineering 40:82-94

Jung G-U, Moon T-H, Park G-W, Lee J-Y, Lee B-H (2013) Use of augmented reality-based training with EMG-triggered functional electric stimulation in stroke rehabilitation Journal of Physical Therapy Science 25:147-151

Kawase T, Sakurada T, Koike Y, Kansaku K (2017) A hybrid BMI-based exoskeleton for paresis: EMG control for assisting arm movements Journal of neural engineering 14:016015

Kiguchi K, Tanaka T, Fukuda T (2004) Neuro-fuzzy control of a robotic exoskeleton with EMG signals IEEE Transactions on fuzzy systems 12:481-490

Kim S, Kim J, Koo B, Kim T, Jung H, Park S, . . Kim Y (2019) Development of an armband EMG module and a pattern recognition algorithm for the 5-finger myoelectric hand prosthesis International Journal of Precision Engineering and Manufacturing 20:1997-2006

Kothe C, Medine D, Boulay C, Grivich M, Stenner T (2012) Lab Streaming Layer (LSL)-A software framework for synchronizing a large array of data collection and stimulation devices The Swartz Center for Computational Neuroscience Software Available online: https://code google.com/archive/p/labstreaminglayer/ (accessed on 29 February 2020)

Kutas M, Donchin E (1980) Preparation to respond as manifested by movement-related brain potentials Brain research 202:95-115

Kwon YD, Shatilov KA, Lee L-H, Kumyol S, Lam K-Y, Yau Y-P, Hui P Myokey: surface electromyography and inertial motion sensing-based text entry in ar. In: 2020 IEEE International Conference on Pervasive Computing and Communications Workshops (PerCom Workshops), 2020. IEEE, pp 1-4 
Ladd GT (1911) Elements of physiological psychology: a treatise of the activities and nature of the mind, from the physical and experimental points of view. C. Scribner's sons,

Murakami EAY (2010) Reaction time and EMG measurement applied to human control modeling Measurement 43:675-683

Pezent E, Israr A, Samad M, Robinson S, Agarwal P, Benko H, Colonnese N Tasbi: Multisensory squeeze and vibrotactile wrist haptics for augmented and virtual reality. In: 2019 IEEE World Haptics Conference (WHC), 2019. IEEE, pp 1-6

Putnam W, Knapp RB Real-time computer control using pattern recognition of the electromyogram. In: Proceedings of the 15th Annual International Conference of the IEEE Engineering in Medicine and Biology Societ, 1993. IEEE, pp 1236-1237

Sigrist R, Rauter G, Riener R, Wolf P (2013) Augmented visual, auditory, haptic, and multimodal feedback in motor learning: a review Psychonomic bulletin \& review 20:21-53

Tsenov G, Zeghbib A, Palis F, Shoylev N, Mladenov V Neural networks for online classification of hand and finger movements using surface EMG signals. In: 2006 8th Seminar on Neural Network Applications in Electrical Engineering, 2006. IEEE, pp 167-171

Viriyasaksathian B, Khemmachotikun S, Kaimuk P, Wongsawat $Y$ EMG-based upper-limb rehabilitation via music synchronization with augmented reality. In: 2011 IEEE International Conference on Robotics and Biomimetics, 2011. IEEE, pp 2856-2859

Young EM, Memar AH, Agarwal P, Colonnese N Bellowband: a pneumatic wristband for delivering local pressure and vibration. In: 2019 IEEE World Haptics Conference (WHC), 2019. IEEE, pp 55-60

Yu H-L, Chase RA, Strauch B (2004) Atlas of hand anatomy and clinical implications. Mosby Incorporated,

Zardoshti-Kermani M, Wheeler BC, Badie K, Hashemi RM (1995) EMG feature evaluation for movement control of upper extremity prostheses IEEE Transactions on Rehabilitation Engineering 3:324-333

Zatsiorsky VM, Li Z-M, Latash ML (2000) Enslaving effects in multi-finger force production Experimental brain research 131:187-195 